ARTICLE

DOI: $10.1038 / s 41467-017-02392-5$

\title{
Integrated nano-opto-electro-mechanical sensor for spectrometry and nanometrology
}

Žarko Zobenica (1) 1, Rob W. van der Heijden', Maurangelo Petruzzella', Francesco Pagliano1, Rick Leijssen², Tian Xia ${ }^{1}$, Leonardo Midolo ${ }^{3}$, Michele Cotrufo (1) ${ }^{1}$, YongJin Cho ${ }^{1}$, Frank W.M. van Otten', Ewold Verhagen ${ }^{2} \&$ Andrea Fiore ${ }^{1}$

Spectrometry is widely used for the characterization of materials, tissues, and gases, and the need for size and cost scaling is driving the development of mini and microspectrometers. While nanophotonic devices provide narrowband filtering that can be used for spectrometry, their practical application has been hampered by the difficulty of integrating tuning and read-out structures. Here, a nano-opto-electro-mechanical system is presented where the three functionalities of transduction, actuation, and detection are integrated, resulting in a high-resolution spectrometer with a micrometer-scale footprint. The system consists of an electromechanically tunable double-membrane photonic crystal cavity with an integrated quantum dot photodiode. Using this structure, we demonstrate a resonance modulation spectroscopy technique that provides subpicometer wavelength resolution. We show its application in the measurement of narrow gas absorption lines and in the interrogation of fiber Bragg gratings. We also explore its operation as displacement-to-photocurrent transducer, demonstrating optomechanical displacement sensing with integrated photocurrent read-out.

\footnotetext{
${ }^{1}$ Department of Applied Physics and Institute for Photonic Integration, Eindhoven University of Technology, P.O. Box 5135600 MB Eindhoven, The Netherlands. ${ }^{2}$ Center for Nanophotonics, FOM Institute AMOLF, Science Park 104, 1098 XG Amsterdam, The Netherlands. ${ }^{3}$ Niels Bohr Institute, University of Copenhagen, Blegdamsvej 17, 2100 Copenhagen, Denmark. Correspondence and requests for materials should be addressed to \& Z. (email: z.zobenica@tue.nl)
} 
$\mathrm{T}$ he increasing demand for optical sensing solutions, including e.g., Raman, fluorescence, and absorption spectroscopy, is driving a large effort toward the miniaturization and integration of spectrometers, which has resulted in a range of mini and microspectrometers ${ }^{1-9}$. Most schemes employ diffraction for the spectral discrimination, which inevitably brings a trade-off between size and resolution. Integrated spectrometer implementations $s^{10-15}$ are mostly based on arrays of filter elements, which limit the resolution, and rely on external detectors, resulting in a much increased packaging complexity and cost. In principle, the combination of a tuneable optical cavity and a photodetector can lead to an extremely compact spectrometer, particularly if the detector is integrated inside the cavity ${ }^{16,17}$. However, for many applications, high resolution is needed under a wide range of incident angles and over a wide spectral range. This can only be achieved by a wavelength-scale cavity combining low optical loss, wide free-spectral range (FSR), and large tuneability. So far, tuneable microcavity detectors have achieved limited resolution ${ }^{17}$ and spectral range ${ }^{16}$. Nano-optomechanical structures, such as photonic crystal (PhC) cavities ${ }^{18,19}$ and microring resonators ${ }^{20-23}$, combine high spectral resolution and large optomechanical coupling, resulting in exquisite sensitivity to nanoscale mechanical motion ${ }^{24}$. This interaction between optical and mechanical degrees of freedom can be used to transduce pmscale mechanical displacements into wavelength shifts and vice versa. It potentially opens the way to a new generation of ultracompact optical sensors, particularly spectrometers, if the required control and read-out can be integrated with the sensing part. The device presented in this paper integrates the tuning, sensing, and read-out within a footprint of only $15 \times 15 \mu \mathrm{m}^{2}$ and provides high-resolution spectra even under a large numerical aperture (NA) illumination, when operated as a tunable filter (spectrometer). We further show that wavemeter measurements of a single laser line or an absorption dip in a broad background are possible with a precision three orders of magnitude better than the optical cavity linewidth. Finally, we demonstrate displacement sensing in the same device, using a fixed laser line as input and integrated detection to transduce the thermal motion of the structure.

\section{Results}

Device design. Figure 1 shows the nano-opto-electro-mechanical system (NOEMS) we employ. Our approach (Fig. 1a) is based on an electromechanically tuneable, double-membrane $\mathrm{PhCs}^{25-27}$, and a low-absorption active material (quantum dots (QDs)). Two identical cavities in the two parallel membranes are evanescently coupled so that the two degenerate cavity modes split into combined symmetric (S) and an antisymmetric (As) modes. The resonant wavelengths strongly depend on the separation $d$ between the membranes, as shown in the simulated tuning curves of Fig. 1b. In the range of $d \sim 200 \mathrm{~nm}$, the optical angular frequency shift per displacement $G_{\omega}=\partial \omega / \partial x$ is in the range of $2 \pi \times$ $37 \mathrm{GHz} \mathrm{nm}^{-1}(\partial \lambda / \partial x=0.2 \mathrm{~nm} \text { per } \mathrm{nm})^{27}$. The lower part of the upper membrane and the upper part of the lower membrane are doped in order to form a p-i-n diode. The distance between the membranes can be controlled by using electrostatic actuation provided by a reverse bias voltage $V_{\mathrm{T}}$ across the $\mathrm{p}$-i-n diode. Compared to in-plane capacitive tuning ${ }^{28-30}$, vertical-actuation offers larger capacitance, resulting in more efficient actuation and smaller footprint. Moreover, it enables the actuation and sensing of out-of-plane motion that is relevant for most nanometrology applications, such as atomic force microscopy. The upper membrane is configured as another $\mathrm{p}$ - $\mathrm{i}-\mathrm{n}$ diode, the n-layer being common to both diodes, see Fig. 1a. A layer of InAs QDs, a

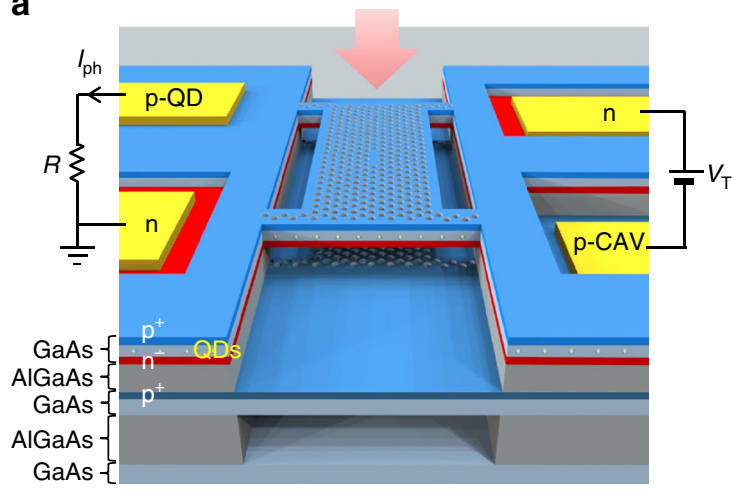

b

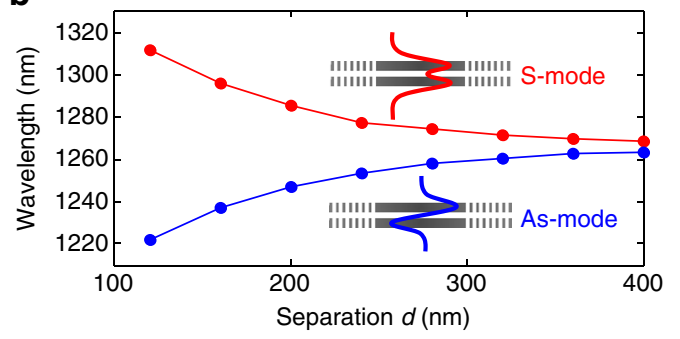

C

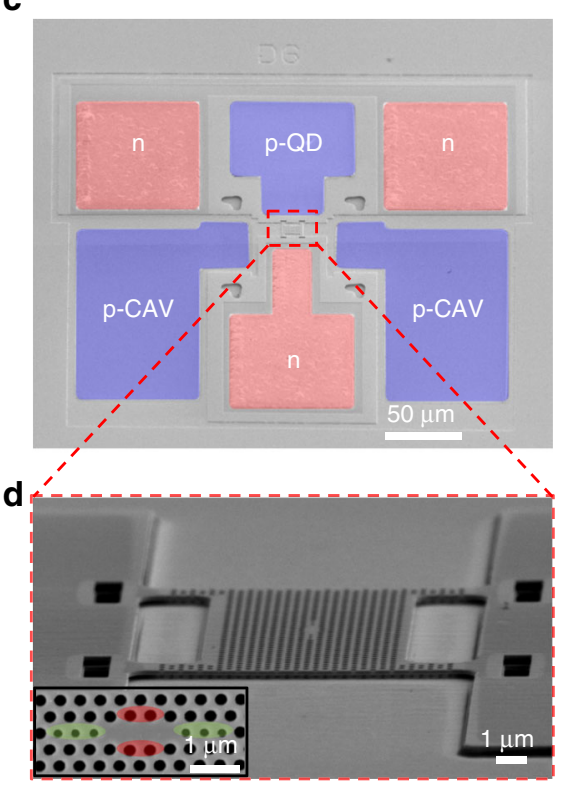

Fig. 1 Overview of the sensor design. a Sketch of the device with electrical contacts and a visible cross-section with p- (blue) and n- (red) doped layers. QDs are located in the middle of the top membrane. Sensor actuation is possible by applying a reverse bias voltage $\left(V_{T}\right)$ to the tuning diode (on the right side), whereas the read-out is done by measuring the photocurrent from the photodiode (left side). $\mathbf{b}$, Simulated optical mode wavelength dependence on the membrane separation for two modes that are symmetric (S) or antisymmetric (As) with respect to the out-of-plane direction. c False-colored SEM image of a typical device (top view) with contact pads to both sensing and actuation diodes. $\mathbf{d}$ Zoom-in SEM image showing the active part of the sensor: a four-arm bridge of dimensions $16 \times 12 \mu \mathrm{m}$ containing a photonic crystal cavity suspended above a fixed photonic crystal membrane. Inset: SEM image of the patterned L3 cavity design modified for high $Q$ factor and large free-spectral range in a double-membrane structure. Optimization was done by displacing horizontally outwards and reducing the radius of six holes (green) and displacing four holes vertically (red) 

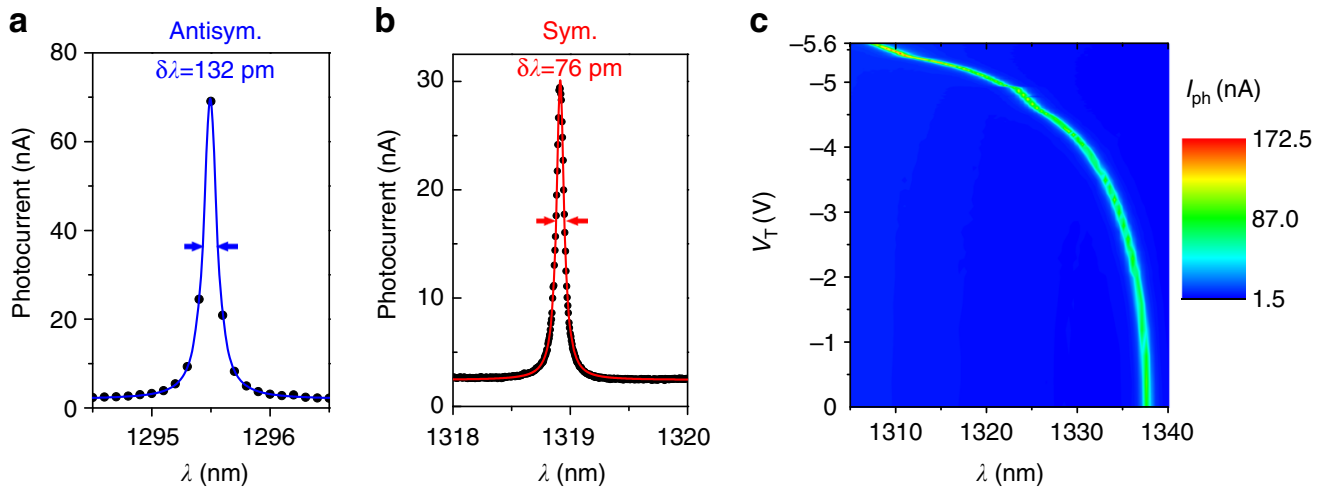

d

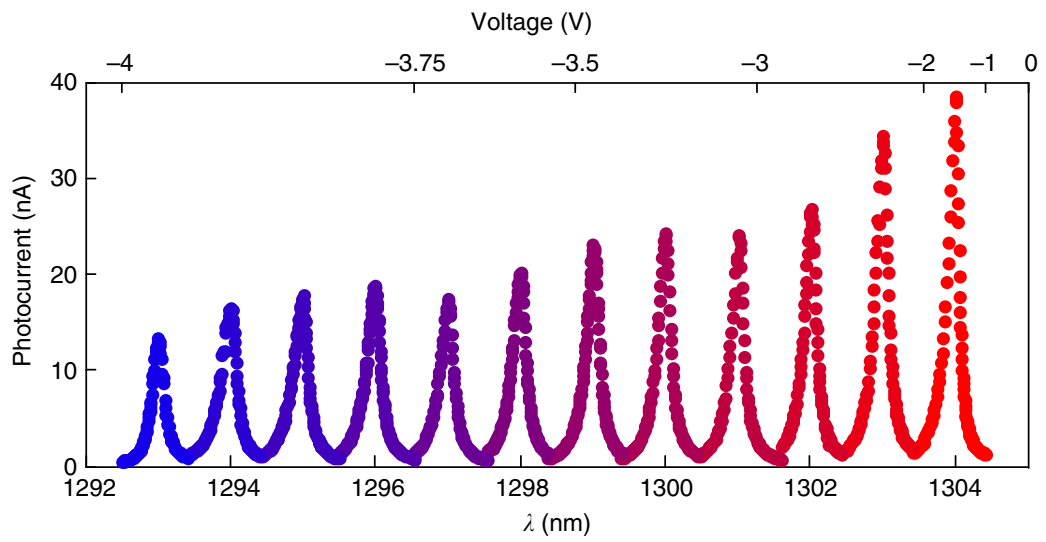

Fig. $2 \mu$-spectrometer measurements. a, b A photocurrent spectrum of the fundamental antisymmetric (symmetric) mode of the three missing holes cavity (L3) modified for high $Q$ factor in double membrane, with a linewidth of $132 \mathrm{pm}(76 \mathrm{pm})$ and a $Q_{\text {factor }}$ of $Q_{A s} \sim 9900$ ( $\left.Q_{S} \sim 17,000\right)$ is shown in blue (red). Data was taken by measuring the photocurrent while a tuneable laser $\left(P_{\text {in }}=125 \mu \mathrm{W}\right)$ was swept across the cavity mode. c Color-coded photocurrent spectra $\left(P_{\text {in }}=25 \mu \mathrm{W}\right)$ showing the fundamental antisymmetric optical modes of an optimized HO cavity spectrally tuned over $30 \mathrm{~nm}$ ( $x$ axis) by increasing the reverse tuning bias $V_{T}$ from 0 to $5.6 \mathrm{~V}$ (y axis) without reaching pull-in. The tuning range is approximately equal to one free spectral range in this case. d Data traces of photocurrent collected by voltage-tuning the optical mode (fundamental antisymmetric mode of the $L 3$ cavity) over a fixed laser wavelength, then changing the laser wavelength by $1 \mathrm{~nm}$ and continuing the voltage tuning. The laser power was coupled into the cavity from top through a 0.45 NA objective, with the power incident on the sample being $12.5 \mu \mathrm{W}$. The scale on the bottom axis in the figure is obtained from a piecewise linear fit of the voltages at the maximum photocurrent versus wavelength. The cavity linewidth provides a spectral resolution of $\sim 200$ pm, and the FSR of $\sim 13$ nm is limited by the crossing with another cavity mode. The decrease in responsivity with decreasing inter-membrane distance (decreasing wavelength) is attributed to an asymmetry in membrane thickness, the upper membrane being $15 \mathrm{~nm}$ thicker $^{46}$

absorbing in the resonant wavelength range, is grown at the center of the upper membrane. The modal absorption, and thereby the detector efficiency and the cavity loss, can be adjusted by controlling the density of the QDs. We chose the dot density so that the absorption contribution to the cavity loss does not limit the $Q$ factor. We estimate the modal absorption ${ }^{31}$ to be $\alpha_{\text {mod }}=2.41 \mathrm{~cm}^{-1}$, corresponding to an absorption-limited quality factor $\left(Q_{\mathrm{abs}}\right)$ of $6.8 \times 10^{4}$. The $\mathrm{PhC}$ cavities are modified L3 or H0 cavities where the position and size of the holes close to the cavity center have been optimized to achieve at the same time a high quality factor and a wide spectral separation (Methods).

Resonant detection. To demonstrate the resonant detection functionality, light from a tuneable laser is coupled into the cavity from the top with a fixed bias on the actuation junction (Fig. 2a, b for the antisymmetric and the symmetric mode, respectively). The photocurrent spectrum shows the cavity resonance, apart from a non-resonant background, and is a result of cavity-enhanced absorption. An experimental cavity linewidth for a symmetric fundamental $\mathrm{PhC}$ mode as narrow as $76 \mathrm{pm}\left(Q_{\mathrm{S}}=1.7 \times 10^{4}\right)$ was obtained utilizing the optimized cavity design from Fig. $1 \mathrm{~d}$ (inset), while corresponding antisymmetric mode is slightly wider, with a linewidth of $132 \mathrm{pm}\left(Q_{\mathrm{As}}=9.9 \times 10^{3}\right)$. Such narrow linewidth corresponds to an order of magnitude improvement over previous reports in resonant cavity detectors ${ }^{17}$. We further investigate the tuneability. As for this particular design, the symmetric cavity mode is located close to the antisymmetric band edge, with several nearby peaks, we consider the antisymmetric mode in the following. As shown in Fig. 2c, blue-tuning of such mode by as much as $30 \mathrm{~nm}$ is obtained for a small applied voltage of $5.6 \mathrm{~V}$, corresponding well to the simulated membrane tuning until the pull-in limit ( $1 / 3$ of the original distance) inherent to capacitive tuning ${ }^{32}$. The tuning range can be extended to $40-50$ $\mathrm{nm}$ if the initial membrane separation in the design is reduced by a factor of two (from 240 to $120 \mathrm{~nm}$ ), and even further if actuation beyond pull-in is realized ${ }^{33}$. The mode used in Fig. $2 \mathrm{c}$ is the fundamental antisymmetric mode of a H0 cavity optimized for high FSR, where we define FSR as the maximum wavelength range for which there is only the mode of interest. Large FSR in this case comes at a price of a larger linewidth of $0.7 \mathrm{~nm}\left(Q_{\exp }=\right.$ $\left.1.9 \times 10^{3}\right)$. The device maps the combination of the incident spectral power density $S(\omega)$ and inter-membrane distance $d$ into a photocurrent signal $I_{\varphi}(d)=R \int_{-\infty}^{\infty} S(\omega) L_{\text {cav }}(d, \omega) \mathrm{d} \omega$, where $R$ is the responsivity $\left(\mathrm{A} \mathrm{W}^{-1}\right)$ and $L_{\text {cav }}(d, \omega)$ the normalized spectral 
a

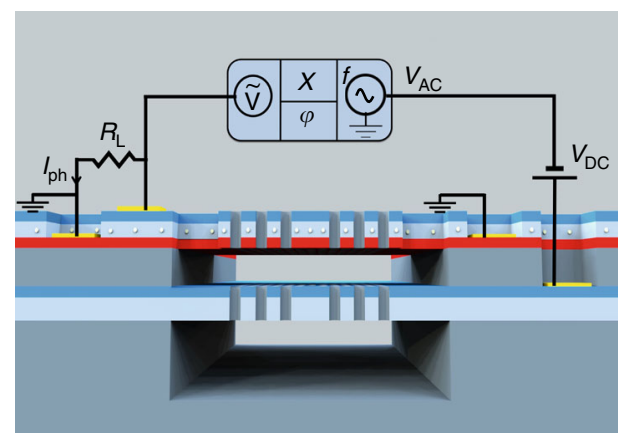

b

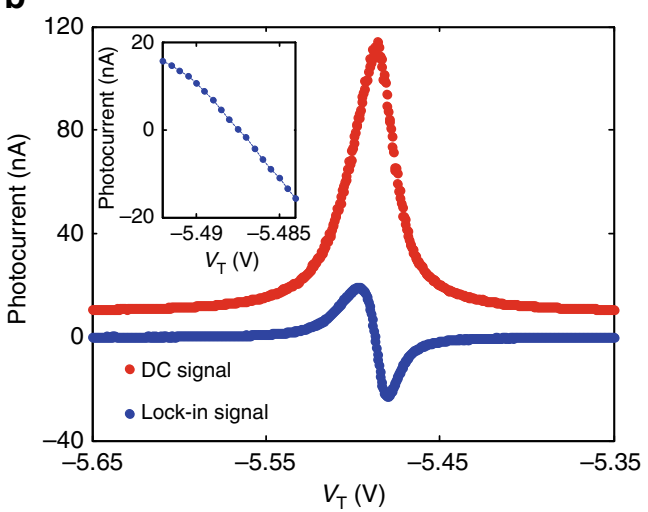

Fig. 3 Resonance modulation spectroscopy. a Sketch of the circuit used for resonance modulation spectroscopy. On the tuning probe input (right side), a modulated signal $\left(V_{A C}\right)$ at frequency $f$ coming from the lock-in is superimposed on a tuneable $D C$ voltage $\left(V_{D C}\right)$. On the detector output (left side), the photocurrent $\left(I_{\mathrm{ph}}\right)$ produces a voltage drop on a load resistor $\left(R_{\mathrm{L}}\right)$ and its in-phase component (X-channel) and its phase $(\varphi)$ at the frequency $(f)$ are measured by the lock-in amplifier. $\mathbf{b}$ Comparison of a laser line recorded using two operation modes of the sensor: spectrometer mode described earlier (red dots) and resonance modulation mode (blue dots). Both measurements were performed simultaneously, by sweeping the tuning voltage and reading the photocurrent DC value (red) and the in-phase component measured by the lock-in amplifier (blue), using a load resistor $R_{\mathrm{L}}=30 \mathrm{k} \Omega$. Since the blue curve is proportional to the derivative of the red curve, the constant background is eliminated. The cavity is modulated with $V_{\mathrm{AC}}=5 \mathrm{mV}$ pp at $f=608 \mathrm{~Hz}$, and excited at a fixed laser frequency of $1322 \mathrm{~nm}$ (the power incident on the sample is estimated to be $125 \mu \mathrm{W}$ ); Inset: zoom-in of the zero crossing in the resonance modulation mode

shape of the cavity resonance, which is centered at frequency $\omega_{0}$ (d). It can therefore be operated to sense either the spectrum of the incident radiation or the mechanical displacement by recording the photocurrent. In the spectrometer mode, the input spectral power density $S(\omega)$ is measured by actuating the membrane separation, $d=d\left(V_{\mathrm{T}}\right)$, and for displacement sensing, the membrane separation $d$ can be deduced from the resonance frequency. The spectrometer operation is demonstrated for a cavity mode where both $Q$ and FSR are sufficiently high (Fig. 2d), which is the case for the fundamental antisymmetric mode (Y1-As) of the modified L3 cavity, with a calculated $Q=1.2 \times 10^{4}$ and FSR $=13 \mathrm{~nm}$. For a number of fixed laser frequencies, a voltage sweep is made across the resonances. Peak positions were taken as calibration points, with which the voltage scale (top) was converted to the wavelength scale (bottom) in Fig. 2d.

The peak photodiode responsivity for the data in Fig. $2 \mathrm{~d}$ is $R \sim 3 \times 10^{-3} \mathrm{~A} \mathrm{~W}^{-1}$ (Methods). It is limited by the small absorptance $\left(\eta_{\mathrm{a}}=0.1\right)$, which can be increased by increasing the dot density without a large influence on the $Q$, as well as unoptimized coupling efficiency $\left(\eta_{c}=0.03\right)$ that can be improved using a side-coupling scheme. The cavity photocurrent peak (Fig. 2b) is superimposed on a non-resonant background caused by light that is directly absorbed in the top membrane. The limited stray light rejection ratio (typically $10-20 \mathrm{~dB}$ ), may be detrimental when a small spectral feature must be measured on a broad background.

Resonance modulation spectroscopy. We introduce a resonance modulation spectroscopy scheme, which can at the same time suppress the effect of background absorption and dramatically increase the spectral peak position resolution. It is based on the small size and built-in actuation functionality of our NOEMS, which enables modulating the mode resonant wavelength at frequencies up to the $\mathrm{MHz}$ range. Applying a small modulation to the tuning voltage as $V_{\mathrm{T}}=V_{\mathrm{DC}}+V_{\mathrm{AC}} \cos \left(2 \pi f_{\mathrm{m}} t\right)$, the cavity frequency $\omega_{\text {cav }}$ is modulated around its central value $\omega_{0}\left(V_{\mathrm{DC}}\right)$ and the photocurrent $\delta I_{\varphi}^{f_{\mathrm{m}}}$ at frequency $f_{\mathrm{m}}$, as measured using a lock-in amplifier (Fig. 3a), becomes:

$$
\begin{array}{r}
\delta I_{\varphi}^{f_{\mathrm{m}}}\left(\omega_{0}\left(d_{0}\right)\right)=R \delta d \int_{-\infty}^{\infty} \frac{\partial L_{\mathrm{cav}}(d, \omega)}{\partial d} S(\omega) \mathrm{d} \omega \\
=R \delta \omega_{\mathrm{m}} \int_{-\infty}^{\infty} \frac{\partial L_{\mathrm{cav}}\left(\omega_{0}, \omega\right)}{\partial \omega_{0}} S(\omega) \mathrm{d} \omega
\end{array}
$$

where $\delta \omega_{m}=\mathrm{G}_{\omega} \delta d$ is the frequency modulation depth (which we assume much smaller than the optical linewidth). Note that $L_{\mathrm{cav}}$ is assumed to be a Lorentzian $L_{\text {cav }}\left(\omega-\omega_{0}\right)$ of constant width, so that $\partial L_{\mathrm{cav}} / \partial \omega_{0}=-\partial L_{\mathrm{cav}} / \partial \omega$. In the limit where $S(\omega)$ is much narrower than the cavity linewidth, $\delta I_{\varphi}^{f_{\mathrm{m}}}\left(\omega_{0}\right)$ is proportional to the derivative of the cavity resonance lineshape. In the opposite limit of a slowly varying input spectrum, $\delta I_{\varphi}^{f_{\mathrm{m}}}\left(\omega_{0}\right)$ is proportional to the derivative of the input spectrum $\left.\frac{\mathrm{d} S}{\mathrm{~d} \omega}\right|_{\omega_{0}}$ as immediately follows from integrating Eq. (1) by parts. The output signal therefore exclusively results from spectral features at the mode frequency and any spectrally flat background is rejected. The principle is demonstrated experimentally for a narrow laser line in Fig. 3b, showing a large improvement of the rejection ratio, from 10 to $27 \mathrm{~dB}$, with values up to $30 \mathrm{~dB}$ measured in other devices. The sign-changing lineshape of the ac photocurrent amplitude also lends itself to the generation of an error signal for feedback-based stabilization. Similarly to frequency ${ }^{34}$ and wavelength ${ }^{35}$ modulation methods, the resonance modulation scheme allows measuring the position of spectral lines with resolution much better than the linewidth-but it can be applied much more widely as it does not require a modulation of the source. From the slope of the derivative curve at the zero crossing (inset Fig. 3b), the voltage-wavelength relation and the measured noise, we calculate a spectral resolution of $100 \mathrm{fm} \mathrm{Hz}^{-1 / 2}$ (Methods), limited by the drift of the cavity resonance during the measurement time. This long-term drift, which produces resonant wavelength shifts in the pm-range over timescales of tens of seconds, is likely related to the adsorption of residual gases on the surface and in the holes of the $\mathrm{PhC}$ and temperature drifts. The 

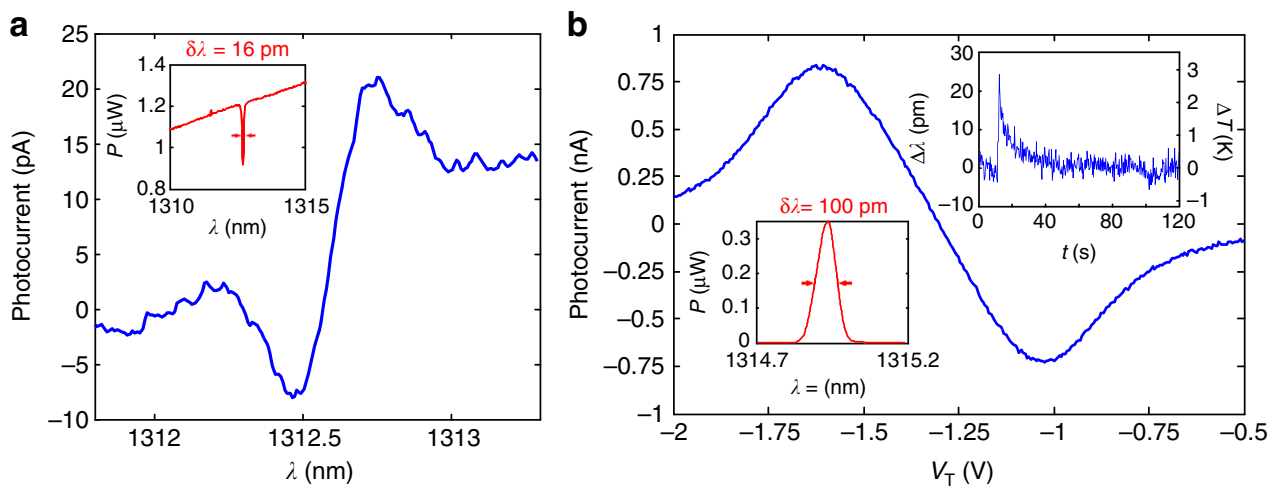

Fig. 4 Applications of resonance modulation spectroscopy. a Gas sensing demonstration, where an HF absorption line at $1312.591 \mathrm{~nm}$ is measured in transmission using the resonance modulation scheme previously described. Voltage was translated into wavelength using a calibration curve obtained from independent PL cavity mode tuning data. A superluminescent diode (SLED) fiber-coupled to an HF gas cell (at $p=50$ Torr), and filtered with a $12 \mathrm{~nm}$ wide $1310 \mathrm{~nm}$ band pass filter is used for excitation. A filter is needed to isolate a single absorption line, and a single cavity mode; inset: optical spectrum analyzer (OSA) spectrum of the SLED with the HF cell inserted, showing the same absorption line. $\mathbf{b}$ Wavemeter measurement of a FBG resonance in reflection performed using the resonance modulation scheme. A SLED is connected to the first input of a $2 \times 2$ fiber beam-splitter with the FBG on one of the outputs, and the second input (reflection) is coupled to the cavity through a NA $=0.45$ objective $\left(P_{\text {in }} \approx 1.6 \mu \mathrm{W}\right)$. The FBG peak (100 pm wide) is read by sweeping a cavity mode $\left(\delta \lambda_{c}=237 \mathrm{pm}, \delta V_{c}=270 \mathrm{mV}\right.$ ), having a sensitivity (slope at zero crossing) of $S_{I}=3.53 \mathrm{nA} \mathrm{V}{ }^{-1}$. The noise measured at the zero crossing is $\delta I_{\text {noise }}=3.6 \mathrm{pA} \mathrm{Hz}^{-1 / 2}$ that translates to a peak wavelength uncertainty of $\delta \lambda_{\text {noise }}=\left(\delta I_{\text {noise }} / \mathrm{S}_{\mathrm{I}}\right) \times\left(\delta \lambda_{\mathrm{c}} / \delta V_{\mathrm{c}}\right)=0.9 \mathrm{pm} \mathrm{Hz} \mathrm{z}^{-1 / 2}$. Inset left: the OSA spectrum of the FBG filter in reflection. Inset right: detuning of the FBG peak (left axis) and corresponding temperature shift (right axis) over a period of $120 \mathrm{~s}$ measured using the cavity sensor. The peak visible at $t=10 \mathrm{~s}$ is induced by convective heating from a heat-gun $50 \mathrm{~cm}$ away from the FBG. The current signal (lock-in output) is translated to displacement $(\Delta \lambda)$ using the slope $S_{1}$. The FBG temperature sensitivity is taken to be $\delta \lambda_{\mathrm{B}} / \delta T=8.5 \mathrm{pm} \mathrm{K} \mathrm{K}^{-1}$ (specified by the manufacturer)

intrinsic resolution, as limited by the electrical noise in the readout, is estimated to be $\sim 2 \mathrm{fm} \mathrm{Hz}^{-1 / 2}$.

Despite the relatively limited spectral range (up to $30 \mathrm{~nm}$ with the present devices, Fig. 2c), these very compact microspectrometers can be employed to monitor specific spectral lines for sensing applications, with outstanding resolution. In order to show their potential in real-world applications, two experiments requiring a high-resolution spectral measurement were conducted: the read-out of a narrow absorption line in transmission (Fig. 4a), and the interrogation of a fiber Bragg grating (FBG) in reflection (Fig. 4b). For the measurement of the absorption line in a broad spectrum, the background rejection provided by the resonance modulation scheme is essential. The experiment is demonstrated in Fig. 4a, where a hydrofluoric acid absorption line is detected, despite the fact that its linewidth $(16 \mathrm{pm})$ is about 15 times narrower than the cavity linewidth used in this experiment $(250 \mathrm{pm})$. The high peak position resolving power of our device also makes it useful for the read-out of temperature, index or pressure sensors based on spectral peak position determination. An example of a FBG peak $\left(\lambda_{\mathrm{B}}=1314.823 \mathrm{~nm}, \delta \lambda_{\mathrm{B}}=100 \mathrm{pm}\right)$ read-out in reflection is presented in Fig. $4 \mathrm{~b}$, with a wavelength resolution (as calculated from the lock-in noise) of $0.9 \mathrm{pm} \mathrm{Hz}^{-\mathrm{I} / 2}$. Without changing the tuning voltage $\left(V_{\mathrm{T}}\right)$, a range of $\sim 500 \mathrm{pm}$ can be covered, and with the tuning it can be extended to $\sim 20 \mathrm{~nm}$. Both resolution and spectral range are comparable to commercial implementations ${ }^{36}$, but they are here obtained with a much smaller footprint (optically active part $15 \times 15 \mu \mathrm{m}$ compared to approximately $\mathrm{cm}$ scale device). The peak position was monitored in time with the NOEMS sensor, and small detunings $\sim 1 \mathrm{pm}$ induced by a short pulse of convective heating $(\Delta T \sim 0.1 \mathrm{~K})$ were succesfully detected (right inset in Fig. $4 \mathrm{~b}$ ). We note that our spectrometer enables the read-out of sensors with very high resolution using a broad spectral source, releasing the need for expensive tuneable lasers. The current implementation can be extended to read a multiplexed input with the use of multiple cavities connected by on-chip waveguides, for multispectral nanometrology applications.
Displacement sensing. To demonstrate the motion sensor functionality, displacement fluctuations due to the Brownian thermal motion of the upper membrane were measured through the photocurrent, which directly monitors the intracavity field. A laser, red-detuned from a high- $Q$ cavity mode (Fig. 5b), is coupled into the cavity and the photocurrent spectrum is measured by an electronic spectrum analyzer (ESA), see Fig. 5a. Transduced thermal motion of the fundamental flexural mode of the top membrane with a frequency $\Omega_{\mathrm{M}} / 2 \pi=2.2 \mathrm{MHz}$ and quality factor $Q_{M} \approx 1400$ is observed, see Fig. 5c. The observed resonant fluctuations can be converted to a displacement spectral density $S_{x x}$ (Fig. $5 c$, right axis) by equating them to the thermal variance $<x_{\text {th }}{ }^{2}>=k_{\mathrm{B}} T / m_{\mathrm{eff}} \Omega_{\mathrm{M}}{ }^{2}$, with $T=297 \mathrm{~K}$ and $m_{\mathrm{eff}}=50 \mathrm{pg}$, obtained from finite element method (FEM) simulations (inset in Fig. 5c) 37. The measurement imprecision is estimated to be $100 \mathrm{fm} \mathrm{Hz}^{-1 /}$ 2 and is presently limited by the pick-up electrical noise in the measurement setup, which produces the noise floor in Fig. 5c. It could be improved to below $1 \mathrm{fm} \mathrm{Hz}^{-1 / 2}$ by optimizing the connection between device and amplifier and increasing the efficiency.

\section{Discussion}

In summary, we have presented the concept of an integrated nanophotonic sensor that embodies the unique features of direct wavelength/displacement detection via photocurrent, and independent voltage control of the optical and mechanical properties of the structure via electrostatic actuation. We demonstrated a high-resolution microspectrometer and proved displacement sensing capabilities on a single device based on coupled $\mathrm{PhC}$ membranes. Furthermore, we introduce a resonance modulation spectroscopy method, exploiting the electromechanical control of the mode wavelength to reject stray light and increase the spectral resolution well beyond the cavity linewidth. Owing to the ultracompact size $\left(15 \times 15 \mu \mathrm{m}^{2}\right)$ of the sensing element, this platform opens the way for mass production of multipurpose highresolution sensors with embedded read-out. As examples of their 
a
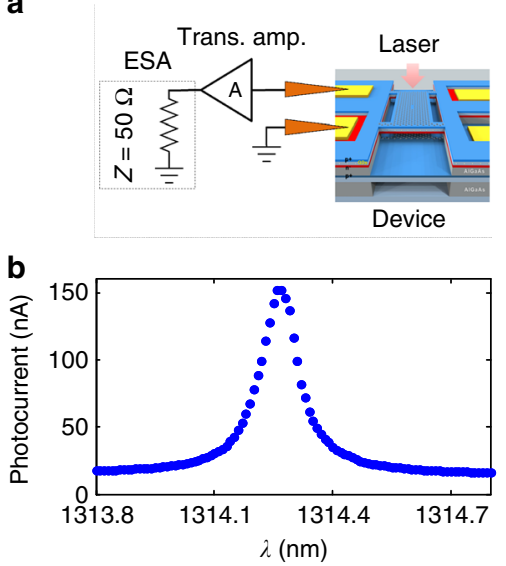

C

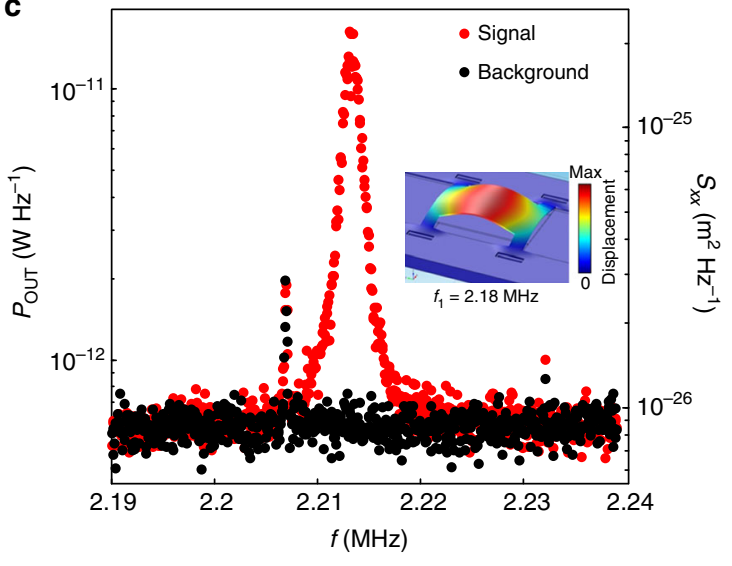

Fig. 5 Brownian motion detected via photocurrent noise. a Sketch of the measurement circuit, with indicated device, input laser, RF probe, amplifier, and the ESA. An RF probe is used to measure the on-chip photodiode, and the signal is sent via an transimpedance amplifier (A in the setup sketch) to the input of the ESA. The device was mounted in a vacuum chamber (pressure below $10^{-4} \mathrm{mbar}$ ). In this experiment, no bias is supplied to the tuning diode. $\mathbf{b}$ Photocurrent measurement of the cavity optical mode for the laser input power $P_{\text {in }}=100 \mu \mathrm{W}$. c ESA spectrum of the photocurrent noise where the fundamental mechanical mode is visible in the output power (red dots) and control measurement with laser off (black dots). The right axis shows the calibrated power spectral density of motion. The CW laser was coupled into the cavity and the laser wavelength was red-detuned from resonance to the wavelength where the photocurrent varies maximally with detuning $\left(\lambda_{\mathrm{L}}=1314.29 \mathrm{~nm}\right)$ and its power was kept low enough so as not to excite selfoscillations $\left(P_{\mathrm{in}}=100 \mu \mathrm{W}\right.$ ). The two other sharp features present in both measurements originate from the environmental RF noise; inset: 3D displacement plot of the 4-arm bridge fundamental mechanical mode with frequency $f_{1}=2.18 \mathrm{MHz}$ simulated using Comsol

potential, we present the measurement of a narrow $(16 \mathrm{pm})$ gas absorption line and the interrogation of a FBG with sub-pm resolution. The sensor concept can be easily applied to other material systems to cover different wavelength ranges (from the visible to the mid-infrared), and further developed for application in temperature, refractive index, and electrical field sensing. The III-V semiconductor platform used for the device could be further exploited to integrate the light source, opening the way to fully integrated optical and optomechanical sensors requiring no external optical connections.

\section{Methods}

Sample structure. The sample was epitaxially grown by molecular beam epitaxy and consists of two GaAs slabs with nominal thicknesses of $170 \mathrm{~nm}$ (bottom) and $185 \mathrm{~nm}$ (top), separated by a $240 \mathrm{~nm}$ thick sacrificial $\mathrm{Al}_{0.7} \mathrm{Ga}_{0.3}$ As layer. A $1.5 \mu \mathrm{m}$ thick $\mathrm{Al}_{0.7} \mathrm{Ga}_{0.3}$ As bottom sacrificial layer separates the membranes from the undoped (001) GaAs substrate. QDs (areal density 70 QDs per $\mu \mathrm{m}^{2}$, with groundstate absorption centered at $1310 \mathrm{~nm}$ at room temperature) are grown in the middle of the upper slab in a Stranski-Krastanov growth process. The upper 70nm-thick part of both membranes was p-doped, while the bottom 70-nm-thick part of the top membrane was n-doped $\left(p_{\text {upper }}=1.5 \times 10^{18} \mathrm{~cm}^{-3}, n=p_{\text {lower }}=2 \times\right.$ $\left.10^{18} \mathrm{~cm}^{-3}\right)$.

Fabrication process and tolerances. The fabrication process begins with defining the vias for the contact pads for the two diodes of the device in two optical lithography steps followed by selective wet and dry etching steps to reach the bottom $\mathrm{p}$ - and the middle n-layer. In the p-via lithography step, four flexible arm bridges, which determine the stiffness of the top membrane, are also defined. To prevent the stress-induced buckling of the bridge, stress-release structures ${ }^{38}$ were implemented (Fig. 1d). No arms are etched in the lower membrane, making it mechanically much less compliant than the upper one. In the third optical lithography step, contact pads for all three doped layers are defined and metals are evaporated. After a lift-off step, $400 \mathrm{~nm}$ of $\mathrm{Si}_{3} \mathrm{~N}_{4}$ is deposited on the sample (hard mask), ZEP resist is spun, and electron-beam lithography (EBL) at $30 \mathrm{kV}$ is performed to define the PhC pattern. After development, the PhC pattern is transferred onto the hard mask using RIE (reactive ion etching) with $\mathrm{CHF}_{3}$. Resist is then removed with oxygen plasma, and the $\mathrm{PhC}$ pattern is imprinted as an array of holes in both membranes using a $\mathrm{Cl}_{2-}$ based inductively coupled plasma (ICP) etching step. Release of the free-standing structure is done by selective wet etching of the sacrificial layer using a cold $\left(1^{\circ} \mathrm{C}\right) \mathrm{HCl}$ solution. To prevent membrane stiction due to capillary forces, supercritical drying in $\mathrm{CO}_{2}$ is employed. Finally, the hard mask is removed by isotropic $\mathrm{O}_{2}-\mathrm{CF}_{4}$ plasma dry etching. The spectral position of the cavities was aligned to the peak of the QDs absorption spectrum by lithographic tuning of the $\mathrm{PhC}$ lattice constant. The QD inhomogeneous broadening (50 nm full width at half maximum in the present sample) could be further increased to reduce the impact of possible QD-cavity spectral misalignment and achieve a wide spectral photocurrent response. The cavity mode position is observed to fluctuate from device to device in a given process in a range of $5 \mathrm{~nm}$ due to various fabrication imperfections: buckling of the top membrane due to residual stress; alteration of the thickness of the top membrane during the dry and wet etchings steps $(<10 \mathrm{~nm})$; changes in the hole radii with respect to design $(<10$ $\mathrm{nm}$ ) and roughness along the hole edges during the ICP etching of the holes; deviations in the lattice constant and position of the holes as determined by the precision of the EBL $(<2 \mathrm{~nm})$. Most of these issues lead to the spectral shift of the modes and/or to a change of the intensity of one of the two types of modes (S or As). The $Q$ factor of the resonances is especially influenced by the ICP etching, hole shape, and roughness, making it the most critical step. We note that all these tolerances are related to the quality of the fabrication process, which could be substantially improved using industrial nanofabrication equipment.

PhC cavity design. The light sensing double-membrane $\mathrm{PhC}$ cavity was designed to ensure small size $\left(V \propto \lambda^{3}\right)$, high $Q$, and large FSR, by modifying standard L3 (with three holes missing in the hexagonal $\mathrm{PhC}^{39}$ ) and $\mathrm{H} 0$ (holes displaced around a position in the lattice ${ }^{40}$ ) designs. In the design of the $\mathrm{L} 3$ cavity used in the experiment in Figs. 2a, b, d, 3, 4 and 5, the position and the radii of the closest six holes in the $x$-direction (displacements $s_{1} / a=0.3, s_{2} / a=0.225, s_{3} / a=0.1$; radii $r_{1}=$ $r_{2}=r_{3}=0.6 \times r$, with $r / a=0.306$, the radius of the holes in the PhC and $a$ the lattice parameter), and the position of the four holes in the $y$-direction $\left(h_{1} / a=0.05\right)$ were optimized, providing a simulated $Q$ factor of $Q_{\text {cold }}=4.4 \times 10^{4}$ for the fundamental symmetric mode (Y1-S) in a cavity without the absorber and a mode spacing of $\Delta \lambda$ $=24 \mathrm{~nm}$ (from 3D FEM simulations). In the case of the fundamental symmetric mode (Y1-S), the experimental result mentioned in the main text (Fig. 2b), provides the $Q$ factor of $Q_{\exp }=1.7 \times 10^{4}$. The absorption losses were estimated to give $Q_{\text {abs }} \approx 6.8 \times 10^{4}$. We attribute the additional loss to scattering losses, patterning errors, and other fabrication imperfections: $Q_{\text {fabr }}=\left(1 / Q_{\exp }-1 / Q_{\text {cold }}-1 / Q_{\text {abs }}\right)^{-1}=$ $4.7 \times 10^{4}$. The $\mathrm{H} 0$ cavity used for the experiment in Fig. $2 \mathrm{c}$ was designed for increased FSR by optimizing the position and radius of four holes. For a triangular lattice with $r / a=0.33$ and parameters $s_{x}=0.15, s_{y}=0.06$, and radii, $r_{x} / r=0.7$ and $r_{y} /$ $r=0.75$, a simulated mode spacing of $\Delta \lambda=30 \mathrm{~nm}$ and $Q$ factor of $1.8 \times 10^{4}$ were obtained.

Responsivity and coupling efficiency. The peak responsivity is given by $R=(e)$ $h \nu) \eta_{\mathrm{c}} \eta_{\mathrm{a}} \eta_{\mathrm{i}}\left(e\right.$ elementary charge, $h$ Planck constant, $\nu$ light frequency, $\eta_{\mathrm{c}}$ coupling efficiency of light into the cavity mode, $\eta_{\mathrm{a}}$ fraction of cavity photons absorbed by the QDs, $\eta_{\mathrm{i}}$ internal efficiency of converting absorbed photons into collected electrons and holes). The internal efficiency is estimated to be close to 1 , as we did not observe any change of photocurrent with applied reverse bias on the detector junction, indicating that carriers are efficiently extracted from the QDs. The 
current responsivity is limited by $\eta_{\mathrm{a}}$ and $\eta_{\mathrm{c}}$. The absorption and thereby $\eta_{\mathrm{a}}$, which is given by $\eta_{\mathrm{a}}=Q_{\text {exp }} / Q_{\mathrm{abs}}$, can be increased by increasing the QD density and number of QD layers, until the point when the absorption losses are comparable to the scattering losses, analogous to designs of Fabry Perot resonant cavity-enhanced photodetectors ${ }^{41}$. The free-space coupling currently employed is expected to have a low efficiency $\eta_{c}$ ( $3 \%$ in the main text) due to the mismatch between the k-vector distribution of the incident field and the one of the cavity mode. The L3 design can be optimized to reach free-space coupling efficiencies up to $15 \%$ while maintaining a high $Q^{42}$, and with grating couplers cavity coupling efficiencies of $20 \%$ have been obtained ${ }^{43}$. It is known that coupling with on-chip waveguides can be very efficient $\left(>50 \%{ }^{44,45}\right)$. Preliminary results of simulations show that similar coupling efficiencies can be expected when coupling waveguides to double-membrane cavities.

Experimental setup. Light from a tuneable laser (Santec TSL-510) was coupled into the cavity from the top through a $\times 50$ objective $(\mathrm{NA}=0.45)$. All laser powers indicated in the main text are values incident on the sample. The two diodes were connected using two adjustable RF probes. In Figs. 2 and 3, the photocurrent was measured as a voltage drop on a $30 \mathrm{k} \Omega$ load resistor ( $\mathrm{R}$ in Fig. 1a). For the measurements in Figs. 4 and 5, the photocurrent was amplified using a transimpedance amplifier $\left(A=5 \times 10^{5} \mathrm{~V} \mathrm{~A}^{-1}\right)$. All measurements were performed at room temperature. The thermal noise measurements in Fig. 5 were performed under vacuum conditions $\left(p<10^{-4}\right.$ mbar) to suppress viscous air damping.

Wavelength resolution in the resonance modulation scheme. The wavelength resolution in Fig. 3b is determined by measuring the current noise in the read-out when laser is on resonance with the cavity, $\delta I_{\text {noise }}=50 \mathrm{pA} \mathrm{Hz}^{-1 / 2}$ (measured using the lock-in-amplifier) and the slope of the derivative curve at the zero crossing $S_{I}=5 \mu \mathrm{A} \mathrm{V}^{-1}$, from which we calculate the voltage accuracy to be $\delta V_{\mathrm{T}}=\delta I_{\text {noise }} / S_{I}=10 \mu \mathrm{V} \mathrm{Hz}^{-1 / 2}$. With the mode wavelength tuning rate being 10 $\mathrm{nm} \mathrm{V} \mathrm{V}^{-1}$, this voltage accuracy can be translated into to a (peak position) resolution of $100 \mathrm{fm}$ when measured in $1 \mathrm{~Hz}$ bandwidth, a value 3 orders of magnitude smaller than the linewidth. As mentioned in the main text, this value is limited by the long-term drift of the cavity resonance. The fundamental noise limit, in the case where no drift is present, would be determined by the thermal noise of the load resistor $\left(\sim 0.7 \mathrm{pA} \mathrm{Hz}^{-1 / 2}\right)$, corresponding to a potential wavelength resolution of $\sim 2$ $\mathrm{fm} \mathrm{Hz} z^{-1 / 2}$, while the photon shot noise $\left(\sim 10 \mathrm{fA} \mathrm{Hz}^{-1 / 2}\right)$ is negligible.

Data availability. All relevant data is available from the corresponding author upon reasonable request.

Received: 31 January 2017 Accepted: 26 November 2017

Published online: 20 December 2017

\section{References}

1. Smit, M. K. \& Van Dam, C. PHASAR-based WDM-devices: principles, design and applications. IEEE J. Sel. Top. Quantum Electron. 2, 236-250 (1996)

2. Sun, Z. J., McGreer, K. A. \& Broughton, J. N. Demultiplexer with 120 channels and 0.29-nm channel spacing. IEEE Photonics Technol. Lett. 10, 90-92 (1998).

3. Horst, F., Green, W. M. J., Offrein, B. J. \& Vlasov, Y. A. Silicon-on-insulator echelle grating WDM demultiplexers with two stigmatic points. IEEE Photonics Technol. Lett. 21, 1743-1745 (2009).

4. Momeni, B., Hosseini, E. S., Askari, M., Soltani, M. \& Adibi, A. Integrated photonic crystal spectrometers for sensing applications. Opt. Commun. 282, 3168-3171 (2009).

5. Kyotoku, B. B. C., Chen, L. \& Lipson, M. Sub-nm resolution cavity enhanced micro- spectrometer. Opt. Express 18, 102-107 (2010).

6. Redding, B., Liew, S. F., Sarma, R. \& Cao, H. Compact spectrometer based on a disordered photonic chip. Nat. Photonics 7, 746-751 (2013).

7. Bao, J. \& Bawendi, M. G. A colloidal quantum dot spectrometer. Nature 523, 67-70 (2013).

8. Shi, Z. \& Boyd, R. W. Fundamental limits to slow-light spectrometers. Opt. Express 21, 7793-7798 (2013)

9. Muneeb, M. et al. Silicon-on-insulator shortwave infrared wavelength meter with integrated photodiodes for on-chip laser monitoring. Opt. Express 22, 27300-27308 (2014).

10. Boutami, S. et al. Highly selective and compact tunable MOEMS photonic crystal Fabry-Perot filter. Opt. Express 14, 3129-3137 (2006).

11. Xia, Z. et al. High resolution on-chip spectroscopy based on miniaturized microdonut resonators. Opt. Express 19, 12356-12364 (2011).

12. Gan, X., Pervez, N., Kymissis, I., Hatami, F. \& Englund, D. A high-resolution spectrometer based on a compact planar two dimensional photonic crystal cavity array. Appl. Phys. Lett. 100, 231104 (2012).
13. Deotare, P. B., Kogos, L. C., Bulu, I. \& Loncar, M. Photonic crystal nanobeam cavities for tunable filter and router applications. IEEE J. Sel. Top. Quantum Electron. 19, 3600210 (2013)

14. Wang, L. H. et al. Realization of optical coupling based on semi-circular photonic crystals. Opt. Int. J. Light Electron Opt. 125, 3641-3645 (2014).

15. Liapis, A. C., Gao, B., Siddiqui, M. R., Shi, Z. \& Boyd, R. W. On-chip spectroscopy with thermally tuned high-Q photonic crystal cavities. Appl. Phys. Lett. 108, 12-16 (2016).

16. Wu, M. S., Vail, E. C., Li, G. S., Yuen, W. \& Chang-Hasnain, C. J. Widely and continuously tunable micromachined resonant cavity detector with wavelength tracking. IEEE Photonics Technol. Lett. 8, 98-100 (1996).

17. Yang, W. et al. Long-wavelength tunable detector using high-contrast grating. IEEE J. Sel. Top. Quantum Electron. 20, 178-185 (2014).

18. Eichenfield, M., Camacho, R., Chan, J., Vahala, K. J. \& Painter, O. A picogramand nanometre-scale photonic-crystal optomechanical cavity. Nature 459, 550-555 (2009).

19. Krause, A. G., Winger, M., Blasius, T. D., Lin, Q. \& Painter, O. A highresolution microchip optomechanical accelerometer. Nat. Photonics 6, 768-772 (2012).

20. Schliesser, A., Del'Haye, P., Nooshi, N., Vahala, K. J. \& Kippenberg, T. J. Radiation pressure cooling of a micromechanical oscillator using dynamical backaction. Phys. Rev. Lett. 97, 243905 (2006).

21. Wiederhecker, G. S., Chen, L., Gondarenko, A. \& Lipson, M. Controlling photonic structures using optical forces. Nature 462, 633-636 (2009).

22. Anetsberger, G. et al. Near-field cavity optomechanics with nanomechanical oscillators. Nat. Phys. 5, 909-914 (2009).

23. Srinivasan, K., Miao, H., Rakher, M. T., Davanço, M. \& Aksyuk, V. Optomechanical transduction of an integrated silicon cantilever probe using a microdisk resonator. Nano Lett. 11, 791-797 (2011).

24. Teufel, J. D., Donner, T., Castellanos-Beltran, M. A., Harlow, J. W. \& Lehnert, K. W. Nanomechanical motion measured with an imprecision below that at the standard quantum limit. Nat. Nanotechnol. 4, 820-823 (2009).

25. Suh, W., Yanik, M. F., Solgaard, O. \& Fan, S. Displacement-sensitive photonic crystal structures based on guided resonance in photonic crystal slabs. Appl. Phys. Lett. 82, 1999-2001 (2003).

26. Notomi, M., Taniyama, H., Mitsugi, S. \& Kuramochi, E. Optomechanical wavelength and energy conversion in high-Q double-layer cavities of photonic crystal slabs. Phys. Rev. Lett. 97, 2-5 (2006).

27. Midolo, L., van Veldhoven, P. J., Dündar, M. A., Nötzel, R. \& Fiore, A. Electromechanical wavelength tuning of double-membrane photonic crystal cavities. Appl. Phys. Lett. 98, 211120 (2011).

28. Winger, M. et al. A chip-scale integrated cavity-electro-optomechanics platform. Opt. Express 19, 24905 (2011).

29. Sun, X., Zhang, X., Poot, M., Xiong, C. \& Tang, H. X. A superhigh-frequency optoelectromechanical system based on a slotted photonic crystal cavity. Appl. Phys. Lett. 101, 221116 (2012).

30. Pitanti, A. et al. Strong opto-electro-mechanical coupling in a silicon photonic crystal cavity. Opt. Express 23, 3196 (2015).

31. Osborne, S. W. et al. Optical absorption cross section of quantum dots. J. Phys Condens. Matter 16, S3749-S3756 (2004).

32. Kovacs, G. T. A. Micromachined Transducers Sourcebook (WCB McGraw-Hill, Boston, 1998).

33. Seeger, J. I. \& Boser, B. E. Charge control of parallel-plate, electrostatic actuators and the tip-in instability. J. Micro. Syst. 12, 656-671 (2003).

34. Black, E. D. An introduction to Pound-Drever-Hall laser frequency stabilization. Am. J. Phys. 69, 79-87 (2001).

35. Bomse, D. S., Stanton, A. C. \& Silver, J. A. Frequency modulation and wavelength modulation spectroscopies: comparison of experimental methods using a lead-salt diode laser. Appl. Opt. 31, 718-731 (1992).

36. Commerical FBG Interrogator. https://www.smartfibres.com/ (2017).

37. Aspelmeyer, M., Kippenberg, T. J. \& Marquardt, F. Cavity optomechanics. Rev. Mod. Phys. 86, 1391-1452 (2014).

38. Iwase, E. et al. Control of buckling in large micromembranes using engineered support structures. J. Micromech. Microeng. 22, 65028 (2012).

39. Chalcraft, A. R. A. et al. Mode structure of the L3 photonic crystal cavity. Appl. Phys. Lett. 90, 241117 (2007).

40. Nomura, M., Tanabe, K., Iwamoto, S. \& Arakawa, Y. Design of a high-Q H0 photonic crystal nanocavity for cavity QED. Phys. Status Solidi 8, 340-342 (2011).

41. Kishino, K. et al. Resonant cavity-enhanced (RCE) photodetectors. IEEE J. Quantum Electron. 27, 2025-2034 (1991).

42. Haddadi, S. et al. High quality beaming and efficient free-space coupling in L3 photonic crystal active nanocavities. Opt. Express 20, 18876-18886 (2012).

43. Chen, Y., Fegadolli, W. S., Jones, W. M., Scherer, A. \& Li, M. Ultrasensitive gasphase chemical sensing based on functionalized photonic crystal nanobeam cavities. ACS Nano 8, 522-527 (2014). 
44. Banaee, M. G., Pattantyus-Abraham, A. G., McCutcheon, M. W., Rieger, G. W. \& Young, J. F. Efficient coupling of photonic crystal microcavity modes to a ridge waveguide. Appl. Phys. Lett. 90, 193106 (2007).

45. Fattah poor, S. et al. Efficient coupling of single photons to ridge-waveguide photonic integrated circuits. Appl. Phys. Lett. 102, 131105 (2013).

46. Petruzzella, M. et al. Nanoscale mechanical actuation and near-field read-out of photonic crystal molecules. Phys. Rev. B 94, 115413 (2016).

\section{Acknowledgements}

This work was supported by the Dutch Technology Foundation (STW), Applied Science Division of NWO, the Technology Program of the Ministry of Economic Affairs under project nos. 12662 and 10380, and by NanoNextNL, a micro and nanotechnology program of the Dutch Ministry of Economic Affairs, Agriculture and Innovations (EL\&I) and 130 partners. It is also part of the research program of the Foundation for Fundamental Research on Matter (FOM), which is financially supported by the NWO. E.V. acknowledges support from an NWO-Vidi grant. The nanofabrication used in this work has been performed in the NanoLab@TU/e cleanroom.

\section{Author contributions}

Ž.Z. designed and fabricated the devices, and performed data analysis. Ž.Z., R.W.v.d.H., F.P., R.L., M.C., and M.P. performed the experiments. T.X., L.M., and M.P. optimized the fabrication recipe. Y.C. and F.W.M.v.O. developed and performed the sample growth. A. F. and R.W.v.d.H. developed the device concept and supervised the project. ŽZ.Z., R.W.v. d.H., E.V., and A.F. wrote the manuscript. All authors discussed the results and commented on the manuscript.

\section{Additional information}

Competing interests: The authors declare no competing financial interests.

Reprints and permission information is available online at http://npg.nature.com/ reprintsandpermissions/

Publisher's note: Springer Nature remains neutral with regard to jurisdictional claims in published maps and institutional affiliations.

\section{(c) (1)}

Open Access This article is licensed under a Creative Commons Attribution 4.0 International License, which permits use, sharing, adaptation, distribution and reproduction in any medium or format, as long as you give appropriate credit to the original author(s) and the source, provide a link to the Creative Commons license, and indicate if changes were made. The images or other third party material in this article are included in the article's Creative Commons license, unless indicated otherwise in a credit line to the material. If material is not included in the article's Creative Commons license and your intended use is not permitted by statutory regulation or exceeds the permitted use, you will need to obtain permission directly from the copyright holder. To view a copy of this license, visit http://creativecommons.org/ licenses/by/4.0/.

(C) The Author(s) 2017 